Running Head: ECOCULTURAL PERSPECTIVE ON FAMILY ACTIVITIES

\title{
Family activities in families including a young child with a significant cognitive and motor developmental delay: an ecocultural perspective
}

Ines Van keer, ${ }^{\mathrm{a},}$, Noor Seghers ${ }^{\mathrm{a}}$, Karla Van Leeuwen ${ }^{\mathrm{a}}$, Bea Maes ${ }^{\mathrm{a}}$

${ }^{a}$ Catholic University of Leuven, Faculty of Psychology and Educational Sciences, Research Unit Parenting and Special Education, Leopold Vanderkelenstraat 32, box 3765, 3000 Leuven, Belgium.

* Corresponding author at: Vanderkelenstraat 32, bus 3765, 3000 Leuven, Belgium.

Tel: +32 16 376892, E-mailaddress: ines.vankeer@kuleuven.be

https://orcid.org/0000-0002-7848-1276

\section{Conflict of interest}

Ines Van keer declares that she has no conflict of interests. Noor Seghers declares that she has no conflict of interests. Karla Van Leeuwen declares that she has no conflict of interests. Bea Maes declares that she has no conflict of interests.

\section{Acknowledgements}

We would like to thank all participating families for their time, effort and enthusiasm. This work was supported by the Research Foundation in Flanders, Belgium [grant ZKC9876] and approved by the Social and Societal Ethics Committee (KU Leuven, Belgium). 
Running Head: ECOCULTURAL PERSPECTIVE ON FAMILY ACTIVITIES

Family activities in families including a young child with a significant cognitive and motor developmental delay: an ecocultural perspective

\begin{abstract}
Background: Families including a young child with a significant cognitive and motor developmental delay need to adapt their routines to the child's needs, while balancing the resources, needs and values of the whole family system. We aim to characterize if and how their routines are sustainable in terms of ecological fit, congruence and meaningfulness, in comparison with families including a typically developing child.

Methods: Based on an adapted version of the Ecocultural Family Interview, conducted with one or both parent(s), the routines of 6 families within the study group and 5 families within the control group were analysed by combining directed content analysis with quantitative ratings.

Results: A myriad of common as well as group-specific indicators of ecological fit, strategies for reaching congruence as well as reported family values were identified.

Conclusions: Parents actively influence family life sustainability. However, families including a child with complex needs are more dependent on others and could benefit greatly from improving the organization of formal support.
\end{abstract}

Keywords: family activities, daily activities, ecocultural theory, severe and multiple disabilities, profound and multiple disabilities, PIMD 


\section{Running Head: ECOCULTURAL PERSPECTIVE ON FAMILY ACTIVITIES}

\section{Introduction}

Participation in the routines of everyday life forms an important context for children's development (Weisner, Coots, Bernheimer, \& Arzubiaga, 1997). More specifically, parents take responsibility for organizing a myriad of home and community experiences, especially during the early childhood period (Guralnick, 2005). These experiences might be developmentally beneficial, not only by reinforcing and extending parent-child interactions, but also by introducing the child to the many demands created by different participants and settings (Guralnick, 2011). However, in organizing daily activities, every family faces the challenge of balancing resources, needs and skills, and of negotiating beliefs about which daily activities are meaningful (Weisner et al., 1997). This is the central tenet in ecocultural theory (Super \& Harkness, 1980; Weisner, 1984; Whiting, 1980). According to this theory, the universal adaptive problem facing families is the construction of a sustainable daily routine. Weisner, Matheson, Coots and Bernheimer (2005) describe: "Ecocultural theory combines social ecology indicators known to influence development (such as basic health, material and social resources,...) with measures of activities and shared cultural meanings. Parents' goals for family life, parenting and development drawn from their cultural niche, are important to understand family ecology. Family daily routines represent the operationalization of what cultures and parents jointly have constructed to achieve these personal and cultural goals. (pp. 46)" Thus, ecocultural theory focuses on the daily family routines in order to understand the processes and activities of family social ecology, for which more structural or categorical variables such as SES or income are only proxies (Weisner et al., 2005). Also, some measures of family environment evaluate the 'goodness' of social ecology for only one individual, e.g. a child with a developmental delay, thereby predetermining the valence of family decisions without considering the relationship to the whole family system (Weisner et al., 2005). However, family adaptations have a collective outcome benefiting or harming each member somewhat differently (Weisner et al., 2005). Sustainability of family life does not refer to individual behavior or general family characteristics, but captures a more enduring project: juggling ongoing demands and meeting long-term goals, which is not the same as coping with crisis and stress (Gallimore, Bernheimer, \& Weisner, 1999; Weisner et al., 2005).

Ecocultural theory proposes that the three most important features with regard to the sustainability of family routines are (1) ecological fit, (2) congruence, and (3) meaningfulness (Weisner et al., 2005). Ecological fit refers to the balance between available resources and constraints or limitations; not necessarily in terms of 'more resources' but in terms of resources that match and support the activities within the family's routine. Congruence refers to a balance between each family member's (sometimes competing) individual needs and abilities. Meaningfulness, in turn, refers 


\section{Running Head: ECOCULTURAL PERSPECTIVE ON FAMILY ACTIVITIES}

to parents organizing their routine in a personally, culturally and morally right way; in line with the personal values and goals of all family members. Longitudinally, a fourth feature can be identified, namely 'stability' or 'predictability'. Sustaining family routines is a dynamic process, requiring adaptation through time. Frequent unpredictable changes do not contribute to sustainability.

Parenting children is inevitably associated with many challenges (Nomaguchi \& Milkie, 2003). To sustain their routines, all families make large and small functional adjustments or accommodations (Gallimore, Coots, Weisner, Garnier, \& Guthrie, 1996), such as switching from full-time to part-time employment, altering the home environment to the child's needs, hiring paid assistance with the domestic or childcare tasks etcetera. The process of accommodating can be conscious as well as unconscious and the purpose of accommodating is to lead a daily life that fits with the families' beliefs and values, the needs of family members and perceived expectations from society (Wilder \& Granlund, 2015). However, in the balancing process of creating and sustaining family life, having a child with disabilities poses extra challenges to families (Wilder \& Granlund, 2015). These challenges can include income constraints, parent's paid and domestic work hours, children's survival and developmental needs etcetera (Gallimore et al., 1996; Luijkx, van der Putten, \& Vlaskamp, 2014). Weisner et al. (2005) found that, in families of young children with a developmental delay, higher levels of sustainability are associated with higher social and personal interconnectedness and lower family workload; however these relationships are not linear: how the families balance their workload with other life domains and use their connections is far more important than the quantity of either aspect.

The specific group of children with significant and multiple disabilities demands intense family accommodations from birth and onwards (Wilder \& Granlund, 2015). However, as Wilder and Granlund (2015) state, this group "is often excluded due to skewness of the sample or just because they are too few" (p. 134). It has been demonstrated that the basic care needs of these children place heavy demands on their parents (Tadema \& Vlaskamp, 2010) and affect their objective time use in all the activities of daily life (Luijkx, van der Putten, \& Vlaskamp, 2017a). In the study of Luijkx, van der Putten and Vlaskamp (2017b), parents appraised the impact of their child with multiple disabilities on their lives both positively and negatively. The majority of parents indicated that raising a child with profound and multiple disabilities helped them understand what should be valued in life and that family members' awareness of other people's needs was strengthened (Luijkx et al., 2017b). "Negatively appraised items mostly concerned time use (extraordinary demands on time, reduction of time with friends and disruption in habits), but were 


\section{Running Head: ECOCULTURAL PERSPECTIVE ON FAMILY ACTIVITIES}

also material in nature, such as the negative impact on finances" (Luijkx et al., 2017b, pp. 5). In one Swedish study of eight children between one and ten years old, results showed complex caregiving needs of the children, frequent relations with professionals and a central caregiving and coordination responsibility of the mothers in all families (Wilder \& Granlund, 2015). Father's involvement, connectedness between parents and the availability of emotional support were found to be linked to the sustainability of family life over the course of two years (Wilder \& Granlund, 2015). With regard to specific activities, we already know that young children with a significant cognitive and motor developmental delay generally experience less diverse activities (with an additional lower frequency of out-of-home activities) and show lower engagement levels compared to typically developing children (Van keer, Van Leeuwen, \& Maes, 2019). Axelsson, Imms and Wilder (2014) point out "the need for the people in the proximal environment of [these children] to act as 'scene-setters', that is, people who arrange opportunities for participation. (pp. 2175)"

This study aims to reveal which factors parents perceive to be important in the construction and sustainment of daily life activities in families including a young child (6-59 months) with a significant cognitive and motor developmental delay, in comparison with families including a typically developing child within the same age range. The main question was if and how their daily life activities are sustainable in terms of (1) ecological fit (cf. the balance between resources and constraints), (2) congruence (cf. congruent with needs and abilities of each family member) and (3) meaningfulness (cf. fit with each family member's goals, values and beliefs). We expected a common set of universal family adaptive concerns, with significant variations due to the child's developmental characteristics (Weisner et al., 2005).

\section{Method}

\subsection{Participants}

Two groups of participants were included: children with a significant cognitive and motor developmental delay (DD-group, $n=6$ ) and typically developing children (TD-group, $n=5$ ). For both groups, we aimed to include children between the chronological age of 6 and 59 months. We did not aim to include children before the age of 6 months because, in the DD-group, clear indications of a significant developmental delay should be present and we wanted to respect the high emotional stress of parents in the first months after birth.

A significant cognitive delay was operationalized using the 'Tandemlijst' (Stadeus, Windey, Vermeir, \& Van Driessche, 1994). In the DD-group, we included children functioning below a quarter of their chronological age, which 
Running Head: ECOCULTURAL PERSPECTIVE ON FAMILY ACTIVITIES

is associated with the description of a profound intellectual disability (Grossman, 1973; Hogg, Foxen, \& McBrien, 1981; Vig \& Sanders, 2007). The Tandemlijst is specifically developed for young children with a developmental delay. It includes the developmental steps and milestones used in early intervention programs. By describing the cognitive developmental domain separately and in detail, the influence of the motor limitations on the estimation of cognitive functioning is minimized as much as possible. A significant motor delay was operationalized using the 'Gross Motor Function Classification System - Expanded \& Revised' (GMFCS-E\&R; Palisano, Rosenbaum, Bartlett, \& Livingston, 2007). The GMFCS is specifically developed for and widely used in research on relatively young children with significant motor limitations and shows high interrater reliability $(\mathrm{G}=.93)$, high test-retest reliability $(\mathrm{G}=.79)$, a positive predictive value of .74 and a negative predictive value of .90 (Wood \& Rosenbaum, 2000). The GMFCS and the GMFCS-E\&R show an almost perfect agreement ( $K=.96$; Gudmundsson \& Nordmark, 2013). Also, the instrument provides descriptions of motor abilities for different age bands, including 0 to 2,2 to 4 and 4 to 6 years. In the DDgroup, we included children functioning at level IV or V (indicating a severe impairment) and, additionally, level III when the child was less than 2 years old (since combining level III, IV and V has a better predictive value at this young age; Gorter, Ketelaar, Rosenbaum, Helders, \& Palisano, 2009). Detailed inclusion criteria are available from the first author upon request. The presence of a significant developmental delay in both the cognitive and the motor domain was regarded as a necessary and sufficient inclusion criterion for the DD-group (Nakken \& Vlaskamp, 2007). Children who only showed a significant delay in one of the two domains were not included. We formulated no criteria regarding the cause of the developmental delay and the presence of additional constraints (sensory disabilities, health problems, comorbid diagnoses such as autism spectrum disorder, etc.). Children who were functioning within the age band corresponding to their chronological age, on the Tandemlijst as well as the GMFCS, were regarded as typically developing and therefore included in the TD-group.

Participants were recruited in the context of a larger project on family activities (Van keer, Van Leeuwen, \& Maes, 2019). In this project, more than 300 hospitals, diagnostic centers, early intervention teams and specialized day care centers were contacted in the Dutch-speaking part of Belgium as well as the Netherlands in order to recruit the DD-group. The TD-group was recruited by randomly selecting 5 day care centers and 5 kindergartens within each of the 5 provinces of the Dutch-speaking part of Belgium as well as within the Brussels Capital Region. Professionals, directors and/or teachers within the contacted organizations were asked (by mail and/or by telephone) to inform potential participants on the project. After filling out a questionnaire on the child's functioning and family activities, 
Running Head: ECOCULTURAL PERSPECTIVE ON FAMILY ACTIVITIES

parents were asked if they were willing to participate in or receive additional information on an interview about the construction and sustainment of the daily life activities within their family. Ten out of the 67 parents in the DD-group and fourteen out of the 52 parents in the TD-group initially showed interest. However, some parents did not respond to further contact attempts or chose not to participate due to time constraints or major life events (i.e. moving, birth of a child). Eventually, our study group consisted of 6 families including a young child (6-59 months) with a significant cognitive and motor developmental delay, while our control group consisted of 5 families including a typically developing child within the same age range. This study is approved by the Social and Societal Ethics Committee (KU Leuven) and informed consent was obtained from parents prior to data collection.

Detailed background information on the children and their family context in both groups is presented in table 1. The participating families were all nuclear families including a mother, a father and one or more children; except for one single mother in the DD-group. The mothers were always present at the interview. In half of the DD-group $(n=3)$ the father was also present, while this was only the case in one TD-family. The group characteristics are generally comparable, although children in the TD-group are somewhat younger ( $M=3.22$ years compared to $M=4.13$ years). This was expected, since we experienced that (parents of) children without disabilities are easier to reach at a young age.

\subsection{Instrument and Procedure}

In this study, we administered a self-developed semi-structured interview, strongly based on the Ecocultural Family Interview (EFI; Weisner et al., 1997). The EFI is a guided conversation with parents or caretakers about how they run their daily routines and how they plan, implement, and sustain family activities. The interview focuses on the primary caretaker(s). The original format of the interview is a mix of conversation, probing questions by the interviewer and preplanned structured questions. For example, parents are asked to walk the interviewer through a typical day, interwoven with probing questions about the family's resources and constraints (cf. the family's 'ecology'; 'ecological fit'), their needs and abilities (cf. 'congruence') as well as their goals, values and preferences (cf. the family's 'culture'; 'meaningfulness'). The original EFI interview also incorporates ten life domains in which families can make adaptations in constructing their routine; based on theory (Gallimore, Weisner, Kaufman, \& Bernheimer, 1989) and a longitudinal study on 102 Euro-American families with children (aged three to four years old) with a developmental delay (Nihira, Weisner, \& Bernheimer, 1994). Specific items within these domains can be scored. Three sustainability 


\section{Running Head: ECOCULTURAL PERSPECTIVE ON FAMILY ACTIVITIES}

items (i.e. the ecocultural principles: ecological fit, congruence and meaningfulness) can be rated on an 8-point Likert scale denoting low (0-2), medium (3-5) and high (6-8) scores.

For this study, we preserved the focus on the three ecocultural principles and incorporated questions on the life domains in which families can possibly make (conscious or unconscious) adaptations to create and sustain their daily routine. More specifically, the administered interview consisted of three major parts, as visualized in Figure 1.

In the first (introductory) part, after loosely asking how the family was doing at the moment (i.e. "Are there any 'current' themes, successes, worries at the moment?"), parents were asked to describe when the family members see each other throughout the day (i.e. during the week as well as weekends) and what activities are done at those times. It was clearly stated that activities are defined broadly (i.e. as a daily life activity in which more than one family member takes part; Axelsson, Granlund, \& Wilder, 2013). Parents' answers were visualized on a paper timeline, incorporating 'routine' or 'recurrent' activities as well as more 'sporadical' or 'exceptional' ones. The paper timeline was further used to guide the next two parts of the interview, since it supported the interviewer as well as the parents to substantiate questions and answers with concrete examples, respectively.

In the second part, parents received a brief and simple introduction on the three ecocultural principles and were explicitly asked how they thought each principle played a role in their family life. We started by stating that the organization of the previously discussed activities can be influenced by many factors; some of which are not under voluntary control and others are the result of conscious or less conscious considerations or decisions. Then, each of the three principles was discussed separately by asking questions on what activities each family member found important (cf. meaningfulness), if the current activities were in accordance with each family member's needs and abilities (cf. congruence) and what resources and constraints they could identify in organizing their daily life (cf. ecological fit). The goal of this part of the conversation was to identify the most salient issues, considerations or desires for change within the family, which is why we did not introduce specific topics or factors ourselves. However, we assumed this part would not result in a fully comprehensive picture.

Therefore we included a third part, in which the EFI domains were specifically questioned to ensure comprehensiveness as well as comparability between families. The ten original EFI domains were summarized into six simple and understandable topics: subsistence base (e.g. finances, work), home and environment (e.g. housing, surroundings), domestic workload (e.g. childcare, chores, administration), connectedness of the family (e.g. feelings of togetherness, support, shared decision making), informal network of the family (e.g. support from friends and 


\section{Running Head: ECOCULTURAL PERSPECTIVE ON FAMILY ACTIVITIES}

family, leisure time) and formal network of the family (e.g. involvement of professionals). These last two domains are a summary of five original domains which included a range of (in)formal resources of parent and child (i.e. services, nondisabled network, disability network, support and information). Also, the original 'diversity' domain that was singularly focused on the level of diversity in the family's available cultural and ethnic models was excluded because the researchers did not expect this to be a highly relevant topic within the Belgian and Dutch community. The different domains were visualized with topic cards, containing key words and pictograms. When topics were previously discussed (cf. second part), they were briefly summarized by the interviewer after which parents could provide supplemental information if necessary.

The interview was concluded by asking if the parent(s) thought we had a comprehensive picture of their daily life or if they wanted to add something further. The interviews took place at home or at another place chosen by the parent(s). The length of the interviews ranged from 82 to 153 minutes in the DD-group $(M=110.00, S D=25.78)$ and from 45 to 111 minutes in the TD-group $(M=64.40, S D=26.62)$. All interviews were conducted, transcribed and analyzed by the first author, who has a master's degree in educational sciences.

\subsection{Data analysis}

Interview transcripts were analyzed using directed content analysis, a research method for the interpretation of the content of text data through the systematic classification process of coding and identifying themes or patterns (Hsieh \& Shannon, 2005). In a directed approach, analysis starts with a theory or relevant research findings as guidance for initial codes. Data that cannot be coded is identified and analyzed later to determine if they represent a new category or a subcategory of an existing code. This type of analysis can be characterized as 'deductive' or 'top-down' (Braun \& Clarke, 2006) and is ideal for interviews starting with open-ended questions, followed by targeted questions about predetermined categories (Hsieh \& Shannon, 2005).

In this specific study, a two-step coding process was applied using Nvivo 12 software. First, the interview transcripts were coded to identify possible indicators of the three ecocultural principles (i.e. ecological fit, congruence and meaningfulness). Second, within the identified interview segments, specific domain codes (i.e. subsistence base, home/environment, domestic workload, connectedness, informal and formal network of the family) were used to classify the family's concrete resources, constraints, needs, abilities, goals, values and preferences. One data-extract could receive several codes across, but also within the two coding categories. After initial coding, by looking at the 


\section{Running Head: ECOCULTURAL PERSPECTIVE ON FAMILY ACTIVITIES}

coding intersections between principle and domain codes, it became clear that the three ecocultural principles diverged with regard to the necessary analyzing strategy. Nearly all interview fragments that were coded as an indicator of 'ecological fit' had also received one or more codes at domain-level, identifying a myriad of resources and constraints in establishing a sustainable family routine that could be further divided into concrete subdomains. However, to obtain a comprehensive picture of family's ecological fit, two additional domains ('individual child factors' and 'individual parent factors') were identified. Interview fragments which were coded as indicators of 'congruence' intersected moderately with concrete domain-codes, but instead of simply classifying needs and abilities within the domains, the preliminary analysis pointed towards different strategies families use to maintain a balance between each family member's (possibly competing) individual needs and abilities. Interview fragments coded as indicators of 'meaningfulness' showed almost no intersection with concrete domain-codes, but did highlight specific goals, values and preferences of the families. Further, to obtain a quantifiable indicator of the sustainability of the daily activities within each participating family, their ecological fit, congruence and meaningfulness was globally rated as 'low', 'medium' or 'high' by the first author.

In order to improve the reliability and validity of this study's results, a member check and several peer debriefings were incorporated into the study's design (Creswell \& Miller, 2000). First, the first author provided each family with a summarized account of the indicators of ecological fit, congruence and meaningfulness within their family life. Parents were asked if they fully recognized their family life in the summary, which was confirmed by all families. Second, another researcher who has extensive experience in the guidance of parents with a child with a disability starting from a family quality of life framework read all interview transcripts and blindly double-rated the family's accounts with regard to the three ecocultural principles. In case of non-agreement, final scores were obtained through consensus rating. This was necessary for 6 out of the 33 scores (unweighted kappa of .66), which indicates substantial interrater agreement (Landis \& Koch, 1977). The second researcher also provided critical feedback on each step in the research process (i.e. the interview procedure, the data analysis, the results as well as the discussion).

\section{Results}

\subsection{Ecological fit}

All families in the TD-group are characterized by a high ecological fit, except for Kevin's family due to the combination of an extremely high workload of the father, low connectedness between the parents and a personal 


\section{Running Head: ECOCULTURAL PERSPECTIVE ON FAMILY ACTIVITIES}

vulnerability of the mother (i.e. mother indicates professionals suspect her to have autism spectrum disorder) for which she doesn't receive sufficient support (see table 2). In the DD-group, more variation in ratings is evident. The low rating of Arthur's family is primarily related to the mother being a single parent: she experienced a burn-out due to the difficult combination of full-time work and full-time care of her 4-year old son, further complicated by a limited social network and a limited wish to involve this network. Three families showed medium ecological fit, indicating that some important constraints (e.g. financial constraints in Ben's family, transition in support network in Eva's family and emotional imbalance and high dependency on formal network in Finn's family) impede a strong and sustainable balance between resources and constraints. The high ratings of Cara's and Daisy's family are influenced by stable resources on different domains such as comfortable working hours and job flexibility, the availability of sufficient informal and formal support as well as a high connectedness between and emotional stability of the parents.

On a more specific level, a myriad of (perceived) resources and constraints that serve as indicators of ecological fit were identified within the participants' interviews (cf. table 3). These indicators will be discussed in terms of domains and subdomains. Some of the subdomains were only mentioned by parents within the DD-group, which is why they are indicated as 'DD-specific' in table 3.

\subsubsection{Subsistence base}

In relation to subsistence base, parents in both groups emphasize the importance of their working hours, work motivation, workplace flexibility and financial situation.

In all families, every parental figure is involved in paid employment. Parents primarily indicate a financial motivation, but are also motivated by the personal satisfaction that is associated with their job. The mother of Eva (DD) explains: “Because there you are just [mother's name] you know, there you're not the 'mother of', and you can just do your own thing and the money is also necessary, period". While all fathers work full-time (either through an employment contract or through self-employment), 4 out of 6 DD-mothers and 2 out of 5 TD-mothers work part-time. In both groups, working part-time is generally motivated by three reasons: having more time with the child(ren), more time for the household and more time for the mother herself. The father of Finn (DD), who expresses the wish to reduce his working hours, describes similar reasons: "all organizational tasks... that is a part that I want more time for and also just more time with Finn and a little more free time, so yes, those are three important things that I want to give up my work for." More spare time has additional benefits: "If I would work part-time, I would also be calmer and by 
Running Head: ECOCULTURAL PERSPECTIVE ON FAMILY ACTIVITIES

rushing myself less, I would also have more energy" (mother of Arthur, DD). Notably, for the four mothers in the DDgroup and the mother of Kevin (TD), part-time work is described as a necessity. For example, the mother of Daisy (DD) states: "If I think about having to work full-time and when I think of me and my husband coming home at 5pm and then do everything, really, I would freak out". To the contrary, the mother of Harry (TD) describes working parttime as a very conscious, positive choice: "I actually decided to work less, to see him grow up, because I want to be there a lot".

In both groups, however more pronounced in the DD-group, parents indicate the need for and the presence of job flexibility in at least one of the partners' work situation and generally experience an understanding and flexible attitude of their employer in case of an emergency. Further, only the parents of Arthur and Ben (DD-group) indicate they experience substantial financial problems. The other parents within the DD-group however do emphasize that their financial stability is highly dependent on the received child-related financial support (cf. 3.1.6).

\subsubsection{Home and environment}

In relation to home and environment, both groups indicate the location of their home as an important factor, while parents within the DD-group additionally mention disability-related adjustments in housing as well as disabilityrelated adjustments in and reactions from the environment.

The location of the family home seems to be important in terms of distance (and thus time and mode of transportation) to other important contexts such as the workplace(s), daytime activity of the child(ren), stores and other facilities in the neighborhood. For the DD-families, the specific characteristics of the house in terms of space (e.g. room to move around with a wheelchair, room for a bed box,...) and specific facilities (e.g. adapted bathroom, sling lift,...) are also extremely important. Therefore, disability-related adjustments in housing are perceived as necessary. Finn's family was obliged to move from a small to a large unit within the residential project they already lived in, while the mother of Arthur indicates she and her son might need to move to a new place in the near future, since "the owner of the current apartment does not allow the bathroom to be adapted". In their turn, the family of Ben, Cara, Daisy and Eva have done or are planning to do large home renovations, to cater to the current but also the future needs of the children. Evidently, these adjustments are associated with high costs, but also with a long planning process (cf. time investment) that is complicated by the often unknown future development of the child. The mother of Daisy 


\section{Running Head: ECOCULTURAL PERSPECTIVE ON FAMILY ACTIVITIES}

explains: "It took us over a year and a half to start building... we took the time to think, to talk with therapists and doctors, we consulted other parents, ... we took the time, in the hope that we have not forgotten anything".

Thus the families' homes are very much (in the process of being) adjusted to the child, however, the broader environment often is not. The mother of Eva states: "Off course, it's a recent phenomenon that parents like us just participate in society, in the past, you just put those children away, but the parents nowadays, you know, my child goes everywhere with us, and you just notice that countries like the Netherlands and Belgium are not quite ready... like the pavements, little steps, very small stairs and things like that. They are not quite ready yet". The mother of Daisy agrees: "There often are special toilets for disabled people, but mostly there is no nursing table. For now, baby care tables are still perfect for Daisy, but for her older sister, that is not so obvious anymore". The absence of disability-related adjustments in the environment poses a challenge for these families, during everyday trips, outings and also on holiday. The mother of Arthur does express satisfaction with some specialized initiatives, such as an adapted swimming pool and a lending service for adapted toys. However, she also indicates that these initiatives are scarce, local and often unknown.

An additional challenge all DD-families encounter are 'the staring looks'. Nearly all parents indicate that they consider this a normal phenomenon, are used to it and aren't held back by it: "It's not because you have a disability, that you can't be looked at" (mother of Daisy). However, the mother of Cara states: "It is not the disability of Cara that holds us back, it is the disability of the people who aren't used to it. They can look, but it is the way they do it sometimes, a pitiful look or laughing, continued staring and turning around, Ijust think it's rude. I don't think people have bad intentions, but it's just... 20 times a day. We also received some bad comments, people asking us 'do you come outside with that?' or telling us that 30 years ago, she would not be alive". Four of the DD-families suggest that the reactions from the environment are influenced by the physical appearance and demeanor of the child, such as having "a beautiful face" (Arthur); not having a deformity "such as schisis" (Eva), looking like "a cute little baby" (Finn) or always "smiling and being happy" (Ben). The mother of Eva also underlines the importance of the parents' attitude (cf. 3.1.8): “I walk just as proudly behind Eva's wheelchair as I walked behind her sister's buggy".

\subsubsection{Domestic workload (including childcare)}

In both groups of families, household chores, childcare, transportation efforts and administrational workload contribute to a busy daily schedule. 
Running Head: ECOCULTURAL PERSPECTIVE ON FAMILY ACTIVITIES

How stressful this domestic workload is experienced, depends heavily on the balanced division of tasks. The domestic workload is experienced as more stressful by parents who often face the daily routines alone, such as the mother of Arthur (DD) who is a single parent as well as the mothers of Cara (DD), Harry (TD) and Kevin (TD), because of the fathers' busy work schedule. Families in which both parents work full-time also experience difficulties in handling the domestic workload, which is why household -and to some extent- childcare are two main motivators for taking up part-time work (cf. 3.1.1). In both groups, nearly all families have hired or wish to hire a housekeeper. What stands out is that, in the TD-group, children can be involved in the household: "If we have to cook, than we take her into the kitchen with us, she can put the potatoes in the pot and...stir in the sauce and such things" (Father of Isabel). In the DD-group, parent's attention for the household and the child are more mutually exclusive.

Even so, while household is an important topic in both groups, childcare is a much more pronounced theme in the DD-families. Taking care of (a) typically developing child(ren) is considered self-evident and 'normal' in relation to the children's young age. However, some of these 'normal' challenges associated with the young age of a child are more enduring in the DD-group, such as planning the daily schedule around nap times but especially the interrupted nights: "We're pretty tired after two bad nights, and yeah, then we really need to come up with emergency schedules, which means that one of us sleeps in another room, with ear plugs and a sleeping pill, to make sure at least one of us can think straight the following day" (Father of Finn, DD). Also, the children in the TD-group often already show some degree of independence, while the children in the DD-group are highly dependent on their parents for every aspect of their care. All parents within the DD-group indicate that taking care of their child takes up a lot of time and is associated with a lot of practical hassle. One major concern of all parents is that they often need specialized material or an extra set of hands: "Bathing her now, that really needs to be done by two people, but if she has her own adapted bathroom with a sling lift in the future, than maybe I can do it on my own" (Mother of Eva, DD). A very important consideration in this regard is the increasing size and weight of the children (cf. 3.1.7), as the mother of Eva explains: "Without something high to change her on, you know, we will find a way, but the problem is, at a certain point, our backs can no longer handle it, you know, you can do everything without materials to help, only it is at the expense of our bodies". Eva's mother even consulted an endocrinologist to explore options to slow down Eva's growth process, to ensure the feasibility of physical caretaking (in- and outdoors) as well as physical contact (cuddling). A second major concern in four of the DD-families is that their daily schedule is dependent on the tube feeding of their child: "The tube feeding, you have to stick to it anyway, you can be a little bit flexible, but not that much, because in the end, 
Running Head: ECOCULTURAL PERSPECTIVE ON FAMILY ACTIVITIES

they have to eat five times a day and if we move up one time, everything moves up and we even have to go to bed later" (Mother of Daisy, DD).

The children's physical dependence on the parent(s) and associated need for specialized materials as well as the tube feeding have a major impact on the family's mobility, as the mother of Arthur (DD) explains: "You have to see when I want to go somewhere, it is a full blown move. With kids, you lose some freedom, that's true, but with other kids you can say 'come on, let's get ready and go', but with him, that's not possible". The mother of Daisy (DD) states: "If I was alone with the two kids, I would not get out of the house", while the mother of Cara says "You really need to find something that you can do on your own. Or always ask someone with you".

In that regard, informal as well as formal help is crucial (cf. 3.1.5 and 3.1.6). Family members and friends often provide support when necessary, however, they are reported to be very apprehensive about temporarily taking over childcare (i.e. babysitting) due to the size and weight of the child (which is a particular concern of grandparents), insecurity about tube feeding as well as medical issues (i.e. possible seizures of Arthur and frequently throwing up by Daisy).

Next to childcare and household, both groups of families have to juggle transportation schedules. These are heavily influenced by the distances between important contexts (cf. 3.1.2) and the presence of several children, especially when siblings' daytime activities are located elsewhere (due to age differences or special needs) and when older siblings are bound to recurrent leisure activities.

To conclude, administration is considered a major, time-consuming part of the domestic workload in the DDgroup, while parents within in the TD-group only occasionally mention this topic. This is primarily related to the DDgroup's experiences in obtaining formal support (cf. 3.1.6).

\subsubsection{Connectedness}

In relation to the family's connectedness, the quality of the coparenting relationship as well as feelings of togetherness are mentioned as an important resource or constraint in family daily life, primarily but not exclusively by parents within the DD-group.

First, the general quality of the coparenting relationship (Feinberg, 2003) seems to be fundamental in sustainable family routines. Parents describe this agreement as having a healthy relationship, characterized by clear communication and shared decision making regarding the child. The parents of Arthur, Ben and Finn (DD) indicate 


\section{Running Head: ECOCULTURAL PERSPECTIVE ON FAMILY ACTIVITIES}

that having a child with a disability has put considerable strain on their relationship, which in the case of Arthur, even resulted in a divorce. The mother of Arthur explains: "For my ex-husband, being a couple is the priority and he is doing everything he can to get that right again, but that means he only has us two in mind and that brings an imbalance, because I only have Arthur in mind". The parents of Ben state that they had "some difficult periods in the beginning, knowing about his syndrome", while the parents of Finn say "We survived, we're still together, but it was a close call". Both families indicate they got and still get through the difficult times by talking about it. Communication is key, as is illustrated by the mother of Eva (DD) describing the relationship with her husband: "We're yin and yang. He keeps me with both feet on the ground, because I always want too much, and I feel he always wants too little, so nowadays we just talk about that".

Because of the young age of the children within these families, they don't actively participate in the family's decision making. However, what is also important is a shared feeling of togetherness as a family. For example, the parents of Ben state they find it important "to spend time together as a family, not everyone in their corner or their own world, but really together", while the mother of Eva says that during a weekend without Eva "they did not feel complete".

Within the TD-group, parental agreement and feelings of togetherness are only briefly mentioned as important and present, except for the family of Kevin. His mother explains how detrimental the lack of communication with her husband is for their relationship and the family's functioning: "When it comes to our family, it is hard to make arrangements with my husband. I cannot turn to him, only for practical things, and even then... The children are constantly caught between our conflicts, and they notice it, it is probably why Kevin's little brother doesn't sleep."

\subsubsection{Informal network of the family}

Families in both groups often mention their informal network as an important resource, primarily in terms of contact with family and friends (and additionally, within the DD-group, other parents who have a child with a disability), practical aid with childcare and leisure activities.

In both groups, contact with family and friends is considered a highly valuable resource. Although the frequency and type of contact varies over families and contact is primarily aimed at simply having an enjoyable time together, family and friends are also an important source of practical and emotional support. All parents in the TDgroup generally describe pleasant relationships with their family and friends, except for the mother of Kevin. She 


\section{Running Head: ECOCULTURAL PERSPECTIVE ON FAMILY ACTIVITIES}

describes a difficult contact with her family(-in-law) and a lack of contact with friends, which she experiences as a major constraint in her (and the whole family's) daily life : "I need social contacts, but it is very hard to keep contact with my friends... I would love to go out with friends and my kids and their kids, but right now I'm losing too much energy with the conflicts at home and that's a shame. In that way, Ifeel very... you know, lonely or isolated." The way in which the DD-families describe their relational experiences with family and friends is also diverse. The parents of Ben indicate they have a small family and are "a bit isolated", because they "never really had the time to go somewhere". The parents of Finn live far away from their family but have a lot of friends close by due to the residential project they live in (i.e. 'co-housing'). Arthur's mother indicates that her friends often come to her house and even bring food, because they know she can't leave the house and she doesn't have time to cook. Daisy's and Eva's parents also indicate strong relationships with their friends; Daisy's mother says: "Because of the fact that family and friends continue to welcome our family completely...that helps a lot". Finally, Cara's mother expresses regret about the way old friends have reacted to the birth of Cara: "In the beginning we only really had our parents. We had a lot of friends who didn't exactly know how to deal with the fact that we had a disabled child or they didn't dare to ask about it so as not to hurt us. Luckily that's over now, the friends who didn't dare... they're not in our lives anymore, but I really think that's a shame. I never thought that would happen".

A specific theme that emerged in interviewing parents within the DD-group, is the value of contact with parents who also have a child with a disability. The mother of Finn explains: "Finding emotional support is hard for me. I think that people who don't have a child with a disability or are not involved professionally don't understand what it's like. In that sense, I experience that with my neighbors who have children, it's different, Ifeel a difference in how the mothers deal with each other, talk about raising a child,... It's different with us... They do want to listen to me, but with mothers who have the same experience, it's really special, even if they live a completely different life, if the child has a completely different disability,.. then there is still more support in a way.. or you at least understand each other better". Parents in a comparable situation can also provide other types of support, as the mother of Arthur states: “There are some dumb practical issues, which make me wonder 'How do other families do that'? I'd like to ask them... But it's not that simple, I don't see other parents from Arthur's school because he goes by bus; and I did get an emailaddress from a parent through a support worker, but how do I even begin contacting them?'. In this regard, the mother of Eva indicates going to conferences or meeting days and joining specific social media groups are a helpful way to gather (or share) practical tips with other parents, although "off course you have to know where to find them". 
Running Head: ECOCULTURAL PERSPECTIVE ON FAMILY ACTIVITIES

Further, in both groups, a specific form of practical support that is often provided by family (and to a lesser extent, friends) is the practical aid with childcare in terms of transportation and babysitting. In both groups, this aid is often crucial for the feasibility of the daily routine. For example, the parents of Cara (DD) say "We can't do it alone, we really need our parents. Otherwise it would be impossible." The mother of Jacob (TD) says the same thing: "If my mother wouldn't help us, it would be a whole lot more difficult, because that limits everything. Yes, that would really limit us". As already explained, informal babysitting arrangements in the DD-group are very challenging (cf. 3.1.3) due to the children's size and weight, possible tube feeding and medical issues. Next to this, parents in the DD-group can be apprehensive themselves to ask for help, particularly from friends. The mother of Arthur explains: "Your friends are your friends, they have their own lives. I don't call on them, but I know if something should happen tomorrow, I can count on them".

In both groups, particularly due to time and energy constraints, only a few parents (i.e. the parents of Finn and the mother of Jacob and Kevin) expand their informal network through a recurrent group-based leisure activity (e.g. going to a sports club). Generally, parents indicate the need for a more flexible leisure activity in terms of time planning (e.g. running) and location (e.g. doing yoga at home). Due to the young age of the children, none of them participate in a recurrent leisure activity, however, some of their older siblings do.

\subsubsection{Formal network of the family}

In both groups, the support received from the child's day care or school, external parenting support and practical aid with childcare are mentioned as valuable formal resources in the organization of the family's daily life. Parents within the DD-group also indicate external child therapy, medical support, financial support and particularly their experiences in obtaining formal support as important factors. Strikingly, within the DD-group, this variety of (desired) formal resources is described extensively and ascribed a crucial role in the family's daily life, while the parents within the TD-group casually mention their child is enrolled in a day care or school and they sporadically seek practical aid or parenting support from a formal source.

Whereas the choice for, and the enrollment in a daytime activity is considered self-evident in the TD-group, parents within the DD-group express a range of considerations. First, next to the obvious role of a day care or school in physically watching and taking care of the children, parents are very conscious about the opportunities of developmental stimulation which the daytime activity can provide. The mother of Eva explains: "We're going to 


\section{Running Head: ECOCULTURAL PERSPECTIVE ON FAMILY ACTIVITIES}

change schools for Eva because, among other things, there's too little... Ifeel that the school she's in now is living in the Middle Ages too much, they run their fixed program every week, every week the same thing and I think they've been doing that for over 20 years. Don't get me wrong, the children are lovingly cared for and they get all the love they need, but it ends there, is what I'm saying". However, because of the scarcity in specialized day care centers or schools, parents are often forced to choose a daytime activity in function of transportation distance instead of quality of care and stimulation.

Parents further indicate that they value exchanging experiences with the professionals, through mutual consultation on different aspects of the child's functioning. The mother of Cara explains: "Like the hitting, she needs to stop doing that and that is something we strive towards together. We're not alone. We succeeded in stopping her from throwing things and we accomplished that together because we talked about it, reacted in the same way and that's important, that's nice, we 're in it together'. Acknowledging the expertise of all involved is crucial in this regard: "That's why it works so well, they listen to us as professionals, as experts, as parents, and we listen very carefully to them as professionals in the sense of experience, knowledge, skill regarding disabled children in general and based on what they see from Finn" (Finn's father). Last but not least, a multidisciplinary way of working is greatly appreciated by the parents. In the parent's opinion, combining the expertise of psychologists, occupational therapists, speech therapists, physiotherapists, doctors, neurologists and so on, increases the quality and attunement of children's developmental stimulation. Of course, this requires in-depth communication between the involved professionals: "The physiotherapist noticed how strange it was she knew nothing about my search for a better adapted wheelchair. Turned out the physiotherapist and occupational therapist don't work on the same days" (Mother of Eva).

From a more practical point of view, the daycare or school can also support parents in introducing, trying out, choosing, adapting and getting refunds for specialized materials. Summarized, the more support the daycare center or school can centralize and coordinate, the more time is saved by the parents and children that would otherwise be spent searching support and attending appointments elsewhere: "It really makes a difference that there's a child neurologist coming to the day care every Friday: now we can just write in his booklet which prescription we need and we get it. Before, like clockwork, every three or four weeks, we had to make and go to an appointment with the neurologist, again and again" (Mother of Ben).

All children within the DD-group receive at least one type of (bi-)weekly external therapy, primarily physiotherapy: "At school, she receives physiotherapy and speech therapy once a week, both for one half hour, but 


\section{Running Head: ECOCULTURAL PERSPECTIVE ON FAMILY ACTIVITIES}

with children like Eva, that's not enough. I don't think she is at the maximum of her abilities. So you need to organize things yourself. I don't want to wake up when she's 10 and regret we haven't done more" (Mother of Eva).

Next to the child's daytime activity and external therapy, a specific type of formal support is provided by medical professionals. Most of the children within the DD-group, even at the age of three or four, already have a long medical history. The health issues of the children within this specific study sample are more or less under control and parents report that the hassle of rushing to countless doctor appointments and the associated insecurity and fear has somewhat stabilized. However, parents emphasize there is significant room for improvement in this domain: the communication between doctors and medical facilities themselves as well as towards parents is often lacking, insensitive or just downright insulting. The family of Ben, who has a rare syndrome, has struggled tremendously to find the right medical care: "In the beginning, we had a lot of doctors, a lot of different places and then we just decided to remove one after the other. Now it is centralized. Every time, we had to explain everything all over again; and then they would not talk to each other and one doctor would prescribe us a medicine and the next day, the neurologist would ask us bewildered why he has to take that. We don't know, we're not doctors". Half of the families describe they have felt insulted by doctors, for example the mother of Cara: "When Cara was born, she was completely denigrated in front of our faces, I still struggle with that, the doctor just says 'that's not good and that's bad and that's a problem', just summing everything up, I just thought 'what on earth is still good about my child?!' I really think doctors should get a crash course in communication". Here also, doctors should view the parents as experts on their child: "I often encounter doctors who don't listen to me and just look at Eva. Then I know I will be there again the next day. One time, one of the doctors said: I'm going to send her up based on what you are saying, because if I only look at her symptoms, I would send you home. And that was nice, because when we got there, she turned out to be dehydrated and she was admitted to the hospital. They know now, if we call, something's really up" (Mother of Eva).

Further, some families report additional parenting support; for example in the form of home visits by a professional support worker. The mother of Arthur and the parents of Finn indicate that the support worker is very helpful 'with anything and everything', from guiding them in playing with their child to filling out forms together. The parents of Ben don't share that positive experience: "It didn't work out the way it should have. Oftentimes, she would come in and say 'I didn't bring that because he cannot do it anyway'. Or she would put something in front of Ben, show it one time, and then expect him to mimic it right away. You have to give him time, it doesn't work like that". The parents of Ben do emphasize the possible value of specific syndrome associations or centers of expertise, but regret 


\section{Running Head: ECOCULTURAL PERSPECTIVE ON FAMILY ACTIVITIES}

how hard they are to find: "When you type Down syndrome or autism, you get a lot of hits. But with rare syndromes, you get nothing. We did go to a conference in America, but that's... financially speaking...”. In the TD-group, only the mother of Kevin indicates she is in need of and has been struggling to find adequate and affordable external support related to her suspected autism spectrum disorder.

Parents within the TD-group also don't report a great need of external practical aid with childcare through formal channels such as a health insurance association or a family association, except for occasionally finding a babysitter (e.g. in case a child is ill on a workday or just to have a night out). In the DD-group, parents indicate it is hard to rely on such initiatives: the availability of a professional babysitter is often limited to daytime and weekdays, most people are apprehensive about taking care of a child with a disability or do not have the right experience (cf. parents need to trust the babysitter), sometimes a doctor's note is necessary to apply for help and even then, it can take one or several days until help can be provided. A few parents indicate the possibility of asking professional caretakers or interns from the child's care facility or school to babysit, however, hiring paid assistance in the form of a personal assistant or a recurrent guest family is valued the most and is or will be used by 4 out of 6 DD-families. The major advantage of hiring paid assistance according to parents is the flexibility, in the sense that the support can swiftly respond to momentary needs as well as grow with the family's changing needs on a longer term. Parents also appreciate the independence: they no longer solely have to rely on family and friends, structurally as well as in case of emergency. However, it is also a matter of taking a leap of faith and entrusting your child to someone else. For example, the mother of Eva points out that “it takes some getting used to, having a stranger in the house". Next to this, hiring paid assistance is expensive, while the application procedure for getting a structural refund is complicated, time-consuming and associated with long waiting lists.

However, parents indicate they are very relieved to live in a country where a lot of financial support is available, as the parents of Cara explain: "When I just gave birth to Cara, the first thing we thought was 'we have to sell our car and our house', wow, that first week we really thought that, and then the doctors came and said 'slow down, fortunately, you live in Belgium. And that's true, we've said that to each other a lot since then". A range of expenses can be (partially) reimbursed in Belgium and/or the Netherlands, such as specialized materials, diapers, car fuel, car insurance and service checks for help in the household, while parents can also apply for increased child allowance, time credit at work, etc. 
Running Head: ECOCULTURAL PERSPECTIVE ON FAMILY ACTIVITIES

To conclude, despite parent's general contentment with support options, their experiences in obtaining formal support are strikingly less positive. Parents unanimously and strongly emphasize that applying for help is extremely time-consuming and cumbersome. Often, families are unaware of all support options and have to tiringly search and ask around themselves. The mother of Daisy explains: "It's crazy what we have to fill out, a hundred times the same thing and then I think 'guys, really, those papers you want are probably lying on the desk of your colleague next door'. It is never just easy. The tube feeding for example, you have to go to the hospital, because if you do it through the pharmacy, you pay 10 times as much, but you have to know it, don't you. Half of the people just go to the pharmacy, they don't know. There are a lot of things you have to search for, ask, ask again,... If you're not constantly working on it, you miss a lot." The parents of Cara summarize: "They know damn well we have a child with a disability, but nobody comes and tells us what support we are entitled to. We know everything through other parents, that's a world that has opened up for us." Here also, centralization is very helpful, as the parents of Finn explain: "In our village, we have a youth and family coach who is really there for us and really works as a bridge between us and the rest of the municipal organization, we can just call or e-mail him and he asks us for the necessary information and then he coordinates everything internally. That is just fantastic." Further, the support is often not flexible in the sense that it is not responsive enough to the -rapidly changing and unpredictable- needs of the child: "By the time something gets approved, he has grown out of it. Also, at this point, we have to choose between a wheelchair or a buggy. At this moment, he's better off with a buggy, but next year, it can be completely different. But when, next year, he is in need of a wheelchair, we'll have to wait three years. That doesn't make sense to me" (Mother of Ben). Next to this, fixed regulations often limit the choice options of parents: "We would've really liked organic tube feeding for Finn. They make it in Germany but it is not registered in the Netherlands. It's very expensive, but they can't make an exception, it's impossible accountancy-wise".

To summarize, the mother of Eva emphasizes you have to be very strong as a parent and always stand your ground: "Sometimes you think... it's like you are a criminal. The people you deal with, it's like you are asking their personal money or something. We don't need pity, but we need a little empathy. We're not asking things because we want them, but it is just plain necessary. I get that the system has been abused in the past, but when you are in the system once, come on, that has to be enough. Half of the time, she gets placed in the wrong category. Come on, she just belongs in the other one, please come to our house, then you can see it, you can hold her, you can feel her. Then you'll know." 
Running Head: ECOCULTURAL PERSPECTIVE ON FAMILY ACTIVITIES

\subsubsection{Individual child factors}

During the interviews, parents in both groups cited a few individual child factors that are perceived as either a resource or constraint in the family's daily life. For both groups, the temperament and behavior of the children as well as their health seem to play an important role, while parents within the DD-group additionally mention the children's size and weight (as previously explained in section 3.1.3).

First, the child's temperament and recurrent behavior has a pronounced impact. For example, the mother of Arthur (DD) explains: "I take him everywhere with me, because he is so easy-going”. The mother of Jacob (TD) shares a similar story: "Our kids go with us everywhere, whether we go hiking or swimming or to the tennis club. They listen well, it's not like we have to stay at home because they do this or that, no". However, the child's behavior can also be a challenge: "Cara's awareness has evolved tremendously over the last year, but so much that she is sometimes overwhelmed. Actually quite fast, so when she's somewhere busy, with too much noise, she freezes and has panic attacks. Then we have to leave immediately" (Mother of Cara, DD). The mother of Daisy (DD) shares this experience: "She'll start to cry really fast, it's often not socially appropriate, and then we really have to weigh our options: where do we go, what don't we do and with whom?".

A second influencing factor is the child's health. The mother of Arthur (DD) says: "I want to go to the swimming pool with him every two weeks. I tried that last year, but he is always so sick and catching a cold that I always had to cancel". Even though health issues are more common in the DD-group, parents within the TD-group can also face this challenge: “Harry's younger brother has been sick a lot, at its peak, I was at the doctor's office three times a week for something different. That's very tiring, physically but also mentally because you're worried about your child". The impact of the children's behavior and health is beautifully summarized by the mother of Daisy (DD): "The fact that the children are happy is something that helps us a lot. We don't have kids who are sad and are in pain the whole time, we don't have to run to the hospital all the time. That's something very important. I think it's totally different when you have a sick or disabled child that you see suffering. The fact is, my children are very limited, but they are happy".

\subsubsection{Individual parent factors}


Running Head: ECOCULTURAL PERSPECTIVE ON FAMILY ACTIVITIES

As individual parent factors, fatigue and parental attitude regarding the family situation were identified as important constraints and/or resources in the family’s daily life.

Fatigue (cf. 3.1.3) plays a role in both groups. For example, the mother of Arthur (DD) says “When I'm tired and a mess, he feels that and I can undertake less with him", while the mother of Kevin explains: "I don't have time for a hobby, I'm always cleaning up and then when I have some time during the day, I am so tired I can't concentrate and just do the things that are strictly necessary".

Parents' attitude regarding their family situation, including their acceptance of having a child with a disability, also has a major impact on family life. The mother of Cara says: "We've never made a big deal out of it, we take her everywhere with us, it's just how Cara is and that is simply the normal course of events, it's simply our life", while the mother of Finn (DD) indicates she is still struggling: "Having a child with a disability, that's different from the picture you had in your head. If I see children playing and Finn can't simply join them, that hits me hard. In the beginning and now still, I'd rather not go to places with a lot of healthy children, because it hurts to see the difference".

\subsection{Congruence}

All families in the TD-group are characterized by high congruence, except for Kevin's family: Kevin's father is very focused on his work, while mother desperately tries to structure the family's routines and feels a great need for more time together as a family (see table 2). As a result, Kevin's mother indicates her children are constantly caught between conflicts: "We try to find a balance, but at this point, it's not ideal for anyone". In the DD-group, there is more variation in ratings. The family of Arthur is characterized by low congruence. Arthur's mother has completely placed her son's needs above those of herself and is overwhelmed by the financial pressure and the associated necessity of full-time work, which is something Arthur's father struggles with: he wants Arthur's mother to focus more on herself and their possible reunion as a couple. The families of Ben and Finn show medium congruence. Ben's parents go to great lengths to accommodate to Ben's needs, but are limited by financial constraints and experience a lack of family and, certainly, couple time. Ben's mother indicates she needs the limited individual time she has to "regain strength", both from the busy daily schedule as from the previous turbulent years with Ben. Finn's parents indicate they are still actively seeking a balance between their and Finn's needs and state it requires a great and conscious effort, every day over and over again. Finn's mother still finds it difficult to accept Finn's disability, while Finn's father is planning to reduce his work hours because he needs more time with his friends, his son and his wife. The families of Cara, Daisy 


\section{Running Head: ECOCULTURAL PERSPECTIVE ON FAMILY ACTIVITIES}

and Eva indicate they are at a point in their family life where they have found a balance between the needs of all family members, including the children's siblings. The mother of Eva says: "I don't think Eva's older sister sees it as a disadvantage. I even think that exactly because she is Eva's sister, more is done with her, because as parents we feel it's not completely fair to her'.

Some general remarks on family’s congruence can be made. Each and every family in both groups expresses that throughout daily life, to some extent, they are confronted with tension between the needs of different family members. The mother of Jesse (DD) summarizes: "Yeah, our shared needs, and then our individual needs... It is a field of tension and that's heavy, always thinking and weighing, which is the most important part and which part now needs to be fed". When talking about family member's needs, 'time' is an important issue. In both groups of families, parents generally indicate that their lives are busy and, ideally, they would want more time together as a family, as a couple, with friends and also some more time for themselves individually. The domain of subsistence base plays an important role in this regard: although parents are intrinsically motivated to work, they also experience a lot of financial pressure to work a significant amount of time, which continuously challenges the work-life balance. Generally, within the available time, parent's priority is taking care of and spending quality time with their children. In that regard, the DD-families certainly feel they are faced with a greater challenge in reaching congruence between family member's needs, due to the children's high care needs and associated time investment. Although in some DD-families this resulted in parents forgetting their own needs, the mother of Cara (DD) nuances: "I think sometimes we forget ourselves and us as a couple, but the children have their thing. But I think that's standard, typical for young families, that children demand all the attention. A lot of people say Cara counts for two, I don't see it that way, we just have two kids and none that counts double. All the busyness and all the attention that goes to the children, I think that's the most normal thing in the world at this point in our lives". In some families, searching for congruence is described as a very conscious and active effort ("It requires a lot of planning, a lot of talking to each other", father of Isabel, TD), while in some families it is perceived as a more natural process ("You just do it", mother of Daisy, DD). What does stand out in DD-families, is the (need for) more explicit and conscious attention for the child's current and future needs, not only in terms of 'quality time', but also in terms of the quality of developmental stimulation at the child's daytime activity, necessary additional therapies, child-specific adjustments in housing, participation in society,... Parents within the DDgroup also indicate that the level of congruence can 'fluctuate'. The mother of Eva explains: "I think there are phases. Last week, this week and next week, the balance tips towards Eva due to doctor's visits, filling out forms, meetings at 


\section{Running Head: ECOCULTURAL PERSPECTIVE ON FAMILY ACTIVITIES}

school and the like. But when the summer holiday is over and everyone is back in their rhythm, then I think there is a nice balance. But it can change very quickly, if Eva gets sick or something like that, we stray from the path again".

On a more specific level, different possible strategies that are (or could be) used by families to maintain a balance between each family member's individual needs and abilities were identified within the participants' interviews.

The first identified strategy is reducing work hours: in most of the families, one partner has consciously taken up or reduced to part-time employment or is planning to do so.

A second possible strategy is creating a balanced division of tasks between the parents. Next to the obvious division of household tasks, some parents in both groups report they sometimes alternate childcare responsibilities to create free time for their partner, primarily by staying at home with the child(ren) while the other parent meets with friends or goes to a leisure activity. A few parents in the DD-group also mention splitting up when their child gets overwhelmed during an outing: “When Eva shows she doesn't like the crowds, he goes home with her while I continue shopping with Eva's sister. We're a team like that. It's the same on holiday. Eva still has to take a nap and he loves reading, so then my husband goes back with her, so she can sleep and then he reads in his book". Important to note is that this type of strategy is not available to single parents, like the mother of Arthur.

A third possible strategy is to deliberately and consciously schedule specific types of 'time'. This sounds like an easy task, but is easily forgotten. Next to time for themselves individually, parents can consciously plan time as a couple: "We deliberately got a subscription in the local cultural center, to see some theatre performances together. I thought if we plan those beforehand, then they're fixed and then we go. If you don't have the tickets lying around, there's always an excuse, being tired or the hassle of finding a babysitter and all that. But now we just plan it and do $i t$ " (Mother of Daisy, DD). To cater to the differential needs of different children, parents sometimes consciously reserve private time for one child. For example, the mother of Harry (TD) states: “During the week, Harry's younger brother is often alone with me when Harry's gone to school, so now and then, I really try... For instance, when I go grocery shopping, I only take Harry with me". This private time can be associated with a specific activity: "Amusement parks, you don't go there with Cara, there's no point, that's just a moment for her little sister. That's too bad, but we'll make it up to her, for instance by going to a sensory room with Cara alone" (Father of Cara, DD). When looking at family time, sometimes parents within the DD-group consciously plan outings or holidays without their child with a significant cognitive and motor developmental delay, because of the practical hassle, the reduced choice options 


\section{Running Head: ECOCULTURAL PERSPECTIVE ON FAMILY ACTIVITIES}

regarding the destination and its impact on the 'relaxing' nature of a trip. From an emotional viewpoint, however, this is not always perceived as a viable option: "I'm not up for it yet, going somewhere without the girls. A single day, yes off course, but a whole week... That might change, but I don't think I would fully be in holiday-mode without them" (Mother of Daisy, DD). The mother of Arthur (DD) shares her reservations on transferring the care for her child to someone else: "I can't just drop him off. If another child says he likes going somewhere, you can drop him off with a peace of mind. But Arthur, he can't communicate if he likes it somewhere, so then I'm stuck with the paranoia, what if they are not nice to him or he is bored and he doesn't like it. He can't tell me. So then I think, never mind, I'll keep him with me".

A fourth possible strategy is to deliberately and consciously make choices regarding (the type of) family activities. For example, DD-parents avoid activities that elicit a lot of practical hassle: "If we go out, it has to be something where we can go to, unload the car and stay for a few hours, without constantly having to load and unload the buggy's. A zoo or a shopping mall for instance. Not a city center." (Mother of Daisy, DD). Further, next to alternating between activities that are enjoyed or preferred by specific family members, parents can also select or adapt activities that cater to the needs of different family members at the same time. The father of Finn (DD) illustrates: "Every week we go swimming. We agreed with the teachers that we can flexibly join the toddler swimming lessons and when we do, I adapt the exercises to Finn myself and it's also no problem when we're late because we wanted to sleep in, we need that flexibility. Afterwards, we go to the water jets and have fun together. Meanwhile, Finn's mother is doing swim laps on her own. And when she's finished, we'll play together in the pool until Finn gets tired of it'. In this regard, a common practice in the TD-group is to organize activities with friends who also have children, since this is enjoyed by the parents as well as the children. Another possibility is to make a critical selection in activities, which is primarily described by the parents of Finn (DD): "We try to give our own needs a place by saying no to things that don't contribute to it. For example, we're very critical towards birthday parties or distant family gatherings. Is that something that really contributes, does that make us happy or is it better if we or one of us stays home to do something else?". To conclude, the mother of Eva (DD) underlines the importance of critically reflecting on (the perception of) the child's needs: "In the beginning, I dragged her into every possible amusement park attraction. Now I'm learning to think more from her perspective instead of mine: does Eva really like it or do I merely want her to like it? I think Eva likes it best just being in the line, amongst the people". 
Running Head: ECOCULTURAL PERSPECTIVE ON FAMILY ACTIVITIES

A fifth possible strategy is finding (and accepting) the right support, although it should be noted that some aspects are not under parent's voluntary control. In the interest of balancing needs, some parents in both groups have sought financial support to reduce working hours or to take a break, for example by taking up parental leave or applying for time credit. Further, finding the right (amount of) support with regard to the domestic workload (including childcare) has a major impact on family's congruence, whether the support is formal, informal, structural or sporadic. As stated in paragraph 3.1.6, families can greatly benefit from a support network that works multidisciplinary and is centralized in terms of location and/or contact persons. To conclude, the mother of Ben indicates the importance of selecting the support that you feel comfortable with: "We constantly got to hear 'he should already be able to do this and this'. So we stopped going. We don't need the extra frustration".

\subsection{Meaningfulness}

All families are characterized by a high meaningfulness (see table 2), except for the family of Arthur (DD), Finn (DD) and Kevin (TD). The first two families achieve a medium score since family life is successfully organized according to child-related values (e.g. good quality care and stimulation), but parents strongly indicate that important values regarding their own individual lives are not sufficiently incorporated. Kevin's family receives a low score, since both parents' values differ vastly, with the mother desperately wanting to be a close-knit family and the father valuing work time over family outings, impeding the mother to pursue further family goals in terms of teaching her children how to be independent and adapting an ecological lifestyle. The analysis thus showed that meaningfulness is connected to the family's congruence, in the sense that logically, parents strongly value that the needs of their child and themselves are being met.

More specifically, when asking parents what is most important in their family's life, two general themes were extensively described by parents in both groups. Firstly, most parents strongly value spending time as a family. The father of Isabel (TD) illustrates: “Despite our extremely busy schedule, I find it important to make time for us together at least once a week, to have real family-time”. A second common theme is trying to avoid that daily life is completely determined by the child(ren). The mother of Cara (DD) explains: "Next to being a mother, I'm still myself".

A couple of themes were almost exclusively described by parents within the DD-group. Firstly, almost all parents indicated their profound wish for their children to be happy. For example, the mother of Daisy states: "Happy children, that's all it needs to be" and the mother of Cara says: 'I just want them to have smiled every day, I want them to be happy". Secondly, DD-parents actively indicate the value of good care and stimulation of their child with a 


\section{Running Head: ECOCULTURAL PERSPECTIVE ON FAMILY ACTIVITIES}

disability. For example, the mother of Arthur states: "What I find really important, is that he is interacted with and is being entertained. That sounds logical and simple, but it isn't. Arthur doesn't complain when you just leave him be". The parents of Finn also illustrate this value: "We really want to build a network surrounding Finn as to reach his full developmental potential, that is something that really characterizes our way of parenting". We can assume that parents within the TD-group share the two abovementioned values, however, these seem to be more implicit. In any case, they do not explicitly state either when asked what they value most in daily life, however, two of the TD-parents did state that "school is important". Further, most of the parents within the DD-group explicitly mention the importance of not letting their daily life be completely guided by the child's disability. Cara's father says: "We just want to do the regular things, just do many activities, as much as possible like a normal family". Ben's father agrees: "Even if there are three bags on my right shoulder and two on my left, we still go". However, making compromises is often inevitable, as the mother of Daisy underlines: “We don't go to a restaurant with her anymore. Some people say we don't have to be concerned about it, but if she's crying all the time... You know, I feel people go to a restaurant to have an enjoyable evening, so it's better if she doesn 't come along". Another specific theme is attention for the child's future, for example by wanting to make sure the child "grows up in normal circumstances" (Father of Ben) or by wanting "to keep the children at home for as long as possible" (Mother of Daisy). Lastly, the mother of Eva underlines the importance of independence and self-management: "Preferably, you want do to everything yourself, because... when you're dependent on other people, you are not in control of your own planning. Hopefully, when we have a flexible personal assistant, we can take more control of our own schedule".

Also, some themes were primarily described by parents within the TD-group. First, all five parents indicate they strongly value that their children learn to play with other children. Secondly, three of the TD-parents also explicitly mention the importance of their children learning to play on their own, particularly linked to limiting screen time. Further, the mother of Gwen indicates it is important to her that all of her children can participate in the leisure activity they like. Lastly, the mother of Kevin strongly values that her children learn how to be independent and adapt an ecological lifestyle.

\section{Discussion}

\subsection{Conclusions}


Running Head: ECOCULTURAL PERSPECTIVE ON FAMILY ACTIVITIES

This study confirms the central tenet in ecocultural theory that every family, including a young child with or without a significant cognitive and motor developmental delay, faces the challenge of balancing resources, constraints, needs and skills, and of negotiating beliefs of what daily activities are meaningful. We expected a common set of universal family adaptive concerns, with significant variations due to the child's developmental characteristics (Weisner et al., 2005). This hypothesis is certainly confirmed: a myriad of common as well as group-specific indicators of ecological fit, strategies for reaching congruence as well as reported family values were identified.

A great amount of constraints are perceived as such because they have a negative influence on available time, money and support. Taking care of a child with a significant cognitive and motor developmental delay therefore is a great challenge, especially when combining this care with (full-time) employment. Ecocultural theory underlines that, despite being confronted with significant constraints, families are not hapless victims and actively shape their family life (Weisner, 1986). The families in this study's sample show they play an active role in obtaining a good ecological fit, congruence and meaningfulness (e.g. parents adapting their work schedule, applying for support, making conscious and deliberated choices regarding activities,...) and the families within the DD-group thus demonstrate that having a child with a significant cognitive and motor developmental delay, although it certainly impedes the process, does not necessarily or directly result in a lower sustainability of family life. Parents seem to adapt their expectations to their circumstances, reflecting the concept of 'homeostasis' (i.e. systems return to equilibrium by making adjustments in their response to shocks; Cummins, 2016 ). However, a few major constraints prove to be very challenging and severely impede the process of creating a sustainable family routine, such as being a single parent, serious financial constraints, low parental connectedness and personal vulnerabilities of the parent such as struggling to accept the child's disability.

In that regard, the analysis showed that in order to obtain a fully comprehensive picture, the focus of the original EFI interview on life domains in which families can make (conscious) adaptations might be too narrow. Particularly parents within the DD-group are often faced with and challenged by factors they cannot (easily) influence (e.g. absence of disability-related adjustments in and negative reactions from the environment, size and weight of the child, ...). This consideration is supported by the necessary addition of two domains in the analysis of ecological fit, i.e. individual child and parent factors.

Also, the results of this study indicate that DD-families, in comparison with TD-families, are more dependent on others in being able to create a sustainable family routine. Due to the complex care needs of the children, however, solely depending on an informal support network is not always feasible. Receiving formal support can be a major 


\section{Running Head: ECOCULTURAL PERSPECTIVE ON FAMILY ACTIVITIES}

resource, although parents can be apprehensive about accepting support and even then, it might take some time getting used to it.

Related to this, the results show that much improvement is desirable in the organization of formal support, a topic clearly associated with a lot of frustration in the interviewed parents. Parents indicate a great need of transparent and respectful communication, (being provided with the) knowledge of all available support options as well as the support options themselves being flexible enough to accommodate to the child's changing needs. Also, in order to reduce the enormous and confusing administrative workload, parents -and the whole family for that matter- would benefit greatly from simple and centralized application procedures. Also, a multidisciplinary way of working, especially by the children's day care center or school, can contribute greatly to a sustainable family routine.

The qualitative analysis shows that the three ecocultural indicators of sustainability are very much interrelated. Firstly, ecological fit is an umbrella concept, summarizing all types of resources and constraints families can experience in organizing daily life. Many of these factors, primarily related to subsistence base, connectedness and available support, are also crucial in achieving a balance between parent's and children's needs, which is defined as congruence. Further, in adults it is challenging to differentiate 'values, goals and beliefs' from 'needs', while it is also hard to identify the 'values, goals and beliefs' of young children (either with or without a developmental delay). That is why in this study, the ecocultural principle of meaningfulness is mainly interpreted from the parental point of view. Off course, this does not exclude that the parents' values are related to the child, as is clear from the analysis: parents often strongly value that the needs of their child are being met.

The conceptual interrelatedness of the three ecocultural principles is confirmed by the interrelatedness of their quantitative ratings: most families achieve the same score on each of the principles. However, for three families within the DD-group the scores diverge. Two families achieve a higher score on meaningfulness in comparison to ecological fit and congruence. In both families, forced to set priorities due to a.o. financial constraints, the parents place the needs of the child above those of themselves, which is detrimental for the family's congruence scores. However, the parents also report that they attach tremendous value to and receive a lot of parental satisfaction from the fact that their child is receiving high quality care and stimulation. In other words, although parents certainly wish for and strive towards an improved balance, the imbalance with regard to congruence is to some extent in line with their parental values and contributes to the meaningfulness of their daily life. One family achieves a lower score on ecological fit in comparison to the other two principles. This is primarily related to the necessary and ongoing transition from a more informal 
support network to a more formal support network, due to the increased size and weight of the child. Despite this great challenge, her parents make sure daily life goes on as usual, enjoy their time together and are confident that they will get through this transition phase.

Next to being a useful research framework, the ecocultural perspective can help practitioners in taking and reflecting on a family-centered approach in the guidance of parents with and without disabilities. As Weisner et al. (1997) state, family-centered assistance must be based on a clear understanding of what families are already doing in their daily routines and how families have already juggled their resources, beliefs and values, and the needs and abilities of individual family members. Also, the framework can be used by or with families themselves to trigger reflection on past and necessary family accommodations.

\subsection{Strengths, Limitations and Future Directions}

The major strength of this explorative study is the ecocultural perspective on family life, incorporating the valence of family decisions for the whole family system instead of a single individual. Although the three ecocultural indicators of sustainability are strongly related, they each still provide a unique perspective on the families' daily life: from identifying a broad constellation of resources and constraints to identifying different strategies families use to maintain a balance between family member's needs and uncovering specific family values according to which daily life is organized. By incorporating a control group, universal as well as DD-specific conclusions can be drawn. Also, combining quantitative indicators with a qualitative analysis further strengthens this study's conclusions, with the first type of analysis providing a clear global overview and facilitating inter- and intra-family comparison, and the latter providing in-depth information as the basis for explaining the inter- and intra- family differences. In this study, the three sustainability indicators were rated globally, using broad scoring categories (low, medium, high) instead of using a 9 point-Likert scale as originally proposed in the EFI. This decision was made since the detailed scoring instructions in the EFI manual did not differentiate beyond the global categories, which impeded reaching sufficient (interrater) reliability. Consequentially, the quantitative ratings do not fully represent the differences between the studied families with regard to ecological fit, congruence and meaningfulness. In this regard, the reliability of the scores was prioritized over a more nuanced quantitative differentiation.

Important to note, both the quantitative and qualitative analysis was based on the information provided by the specific interviewees, which did not always include both parents and never included the child(ren), inevitably resulting 
in a second-hand estimation of some family members' perspectives and opinions. Future research could try to incorporate the views of all family members, to obtain a fully representative picture of family life.

Further, Belgium and the Netherlands are characterized by a similar cultural context, however, caution is warranted when interpreting this study's results in the context of other countries. The study's generalizability is also impeded by the rather small sample size and the non- and semi-randomized sampling method, used for the recruitment of the DD- and TD-group respectively. Since we primarily recruited participants indirectly through professionals, we were probably not able to reach (younger) children that stay at home full-time and our insight into a possible selection bias as well as the reasons behind non-participation is fairly limited. It is possible that certain groups of families were not inclined to participate in this study (i.e. due to time constraints, lower interest in or reluctance to talk about the study's topic, ...). The variability in background factors and quantitative ratings as well as the substantial overlap in relevance of the identified factors for all families is reassuring in this regard. However, in future research, it might be interesting to check the relative relevance and exhaustiveness of the identified factors in a larger and more randomized group of participants.

Also, it is important to keep in mind that the study's conclusions are based on the accounts of families including a young child between approximately 2.5 and 4 years old, which is a very specific time in these families' lives. Within the DD-group, many parents indicate their lives have somewhat stabilized after a very turbulent period associated with countless appointments, major insecurities about the child's health condition and emotional challenges related to the child's diagnosis. In this regard, some of the parents also indicate that slowly, but surely, they feel the time has come to start thinking more about their own needs again, even though simultaneously thinking about their child's future (needs). This is an important consideration, because specific age ranges of the child (as well as of parents and siblings) and, possibly associated, transition phases can influence the sustainability of a family's daily life. In line with the theoretically proposed fourth indicator of sustainability, namely 'stability' or 'predictability', longitudinal research into the daily lives of families including a child with a significant cognitive and motor developmental delay is warranted. As Weisner et al. (2005) state: "Parents [continually] reshape their daily family routine to accommodate to their child with delays. This is an achievement worthy of our respect and worthy of scientific inquiry".

\section{Compliance with Ethical Standards}

Funding: This study was funded by Research Foundation in Flanders, Belgium [grant ZKC9876]. 


\section{Running Head: ECOCULTURAL PERSPECTIVE ON FAMILY ACTIVITIES}

Ethical approval: All procedures performed in this study were in accordance with the ethical standards of the institutional research committee and with the 1964 Helsinki declaration and its later amendments or comparable ethical standards.

Informed consent: Informed consent was obtained from all individual participants included in the study.

\section{References}

Axelsson, A. K., Granlund, M. \& Wilder, J. (2013). Engagement in family activities: a quantitative, comparative study of children with profound intellectual and multiple disabilities and children with typical development. Child: Care, Health and Development, 39(4) 523-534.

Axelsson, A. K., Imms, C., \& Wilder, J. (2014). Strategies that facilitate participation in family activities of children and adolescents with profound intellectual and multiple disabilities: Parents' and personal assistants' experiences. Disability and rehabilitation, 36(25), 2169-2177.

Braun, V., \& Clarke, V. (2006). Using thematic analysis in psychology. Qualitative research in psychology, 3(2), $77-$ 101.

Creswell, J.W., \& Miller, D.L. (2000). Determining validity in qualitative inquiry. Theory into Practice, 39, $124-130$.

Cummins, R. A. (2016). The theory of subjective wellbeing homeostasis: A contribution to understanding life quality. In A Life Devoted to Quality of Life (pp. 61-79). Springer, Cham.

Feinberg, M. E. (2003). The internal structure and ecological context of coparenting: A framework for research and intervention. Parenting: Science and Practice, 3(2), 95-131.

Hogg, J., Foxen, T., \& McBrien, J. (1981). Issues in the training and evaluation of behaviour modification skills for staff working with profoundly retarded multiply handicapped children. Behavioural Psychotherapy (New Series), 9(4), 345-357.

Hsieh, H. F., \& Shannon, S. E. (2005). Three approaches to qualitative content analysis. Qualitative health research, 15(9), 1277-1288.

Gallimore, R., Coots, J., Weisner, T., Garnier, H., \& Guthrie, D. (1996). Family responses to children with developmental delays II: Accommodation intensity and activity in early and middle childhood. American Journal on Mental Retardation, 101, 215-232. 


\section{Running Head: ECOCULTURAL PERSPECTIVE ON FAMILY ACTIVITIES}

Gallimore, R., Bernheimer, L. P., \& Weisner, T. S. (1999). Family life is more than managing crisis: Broadening the agenda of research on families adapting to childhood disability. In: Gallimore, R., Bernheimer, L. P., MacMillan, D. L., Speece, D. L., \& Vaughn, S. (Eds.), Developmental Perspectives on children with highincidence disabilities (pp. 55-80). LEA Press, Mahwah, N.J.

Gallimore, R., Weisner, T. S., Kaufman, S. Z., \& Bernheimer, L. P. (1989). The Social Construction of ecocultural niches: Family accommodation of developmentally delayed children. American Journal of Mental Retardation, 94 (3), 216-230.

Gorter, J. W., Ketelaar, M., Rosenbaum, P., Helders, P. J., \& Palisano, R. (2009). Use of the GMFCS in infants with CP: the need for reclassification at age 2 years or older. Developmental Medicine \& Child Neurology, 51(1), 46-52.

Grossman, H. J. (1973). Manual on Terminology and Classification in Mental Retardation. American Association on Mental Deficiency: Special Publication Series, No. 2. Washington D.C.: American Association on Mental Deficiency.

Gudmundsson, C., \& Nordmark, E. (2013). The agreement between GMFCS and GMFCS-E\&R in children with cerebral palsy. The European Journal of Physiotherapy, 15(3), 127-133.

Guralnick, M. J. (2005). Early intervention for children with intellectual disabilities: Current knowledge and future prospects. Journal of Applied Research in Intellectual Disabilities, 18(4), 313-324.

Guralnick, M. J. (2011). Why early intervention works: A systems perspective. Infants and young children, 24(1), 628.

Landis, J. R., \& Koch, G. G. (1977). The measurement of observer agreement for categorical data. Biometrics, 33 (1), $159-174$.

Luijkx, J., van der Putten, A. A. J., \& Vlaskamp, C. (2014). The impact of rearing a child with PIMD: research into time use of parents. Journal of applied research in intellectual disabilities, 27(4), 358-358.

Luijkx, J., van der Putten, A. A. J., \& Vlaskamp, C. (2017a). Time use of parents raising children with severe or profound intellectual and multiple disabilities. Child: care, health and development, 43(4), 518-526.

Luijkx, J., van der Putten, A. A., \& Vlaskamp, C. (2017b). A valuable burden? The impact of children with profound intellectual and multiple disabilities on family life. Journal of Intellectual \& Developmental Disability, DOI: $10.3109 / 13668250.2017 .1326588$ 


\section{Running Head: ECOCULTURAL PERSPECTIVE ON FAMILY ACTIVITIES}

Nakken, H., \& Vlaskamp, C. (2007). A need for a taxonomy for profound intellectual and multiple disabilities. Journal of Policy and Practice in intellectual Disabilities, 4(2), 83-87.

Nihira, K., Weisner, T. S., \& Bernheimer, L. P. (1994). Ecocultural assessment in families of children with developmental delays: Construct and concurrent validities. American Journal of Mental Retardation, 98 (5), $551-566$.

Nomaguchi, K. M., \& Milkie, M. A. (2003). Costs and rewards of children: The effects of becoming a parent on adults' lives. Journal of marriage and family, 65(2), 356-374.

Palisano, R., Rosenbaum, P., Bartlett, D., \& Livingston, M. (2007). Gross Motor Function Classification System: Expanded and Revised. CanChild Centre for Childhood Disability Research, McMaster University.

Super, C., \& Harkness, S. (eds.). (1980). Anthropological perspectives on child development: New directions for child development, No. 8. Jossey-Bass, San Francisco

Stadeus, A., Windey, K., Vermeir, G., \& Van Driessche, S. (1994). Tandemlijst voor jonge kinderen met ontwikkelingsmoeilijkheden. Leuven: Garant.

Tadema, A. C., \& Vlaskamp, C. (2010). The time and effort in taking care for children with profound intellectual and multiple disabilities: A study on care load and support. British Journal of Learning Disabilities, 38(1), 41-48.

Van keer, I., Van Leeuwen, K., \& Maes, B. (2019). Exploring family activities and child engagement: A study on children with a significant cognitive and motor developmental delay. Journal of Applied Research in Intellectual Disabilities. Advance online publication. DOI: 10.1111/jar.12568

Vig, S., \& Sanders, M. (2007). Assessment of Mental Retardation. In Brassard, M. R., \& Boehm, A. E. (Eds.) Preschool assessment: Principles and practices. Guilford Press.

Weisner, T. S. (1984). Ecocultural niches of middle childhood: A cross-cultural perspective. In: Collins, W. A. (ed.), Development during middle childhood: The years from six to twelve, National Academy of Sciences Press, Washington, D.C., pp. 335-369.

Weisner, T. S. (1986). Implementing new relationship styles in conventional and nonconventional families. In Hartup, W. \& Rubin, Z. (Eds.), Relationships and development (pp. 185-206), Hillsdale, N.J.: LEA Press.

Weisner, T. S., Coots, J. J., Bernheimer, L. P., \& Arzubiaga, A. (1997). The ecocultural family interview manual. Los Angeles: UCLA Center for Culture and Health. 
Running Head: ECOCULTURAL PERSPECTIVE ON FAMILY ACTIVITIES

Weisner, T. S., Matheson, C., Coots, J., \& Bernheimer, L. P. (2005). Sustainability of daily routines as a family outcome. In Maynard, A. E. \& Martini, M. I. (Eds.), Learning in cultural context (pp. 41-73). Springer, Boston, MA.

Whiting, B. (1980). Culture and social behavior: A model for the development of social behavior. Ethos, 8, 95-116.

Wilder, J., \& Granlund, M. (2015). Stability and change in sustainability of daily routines and social networks in families of children with profound intellectual and multiple disabilities. Journal of Applied Research in Intellectual Disabilities, 28(2), 133-144.

Wood, E., \& Rosenbaum, P. (2000). The gross motor function classification system for cerebral palsy: a study of reliability and stability over time. Developmental Medicine and Child Neurology, 42, 292-96. 


\section{Running Head: ECOCULTURAL PERSPECTIVE ON FAMILY ACTIVITIES}

Table 1

Overview of the participating families

\begin{tabular}{|c|c|c|c|c|c|c|c|}
\hline & \multicolumn{2}{|c|}{ Child } & \multicolumn{5}{|c|}{ Family } \\
\hline & Gender & Age & Additional family members & Parental education & Parental employment & Country & Interviewee \\
\hline DD-group & & & & & & & \\
\hline Arthur & Boy & 4.15 years & 1 parent & M: higher ed. & M: full-time & $\mathrm{BE}$ & M \\
\hline Ben & Boy & 3.77 years & 2 parents & $\begin{array}{l}\text { M: secondary ed. } \\
\text { F: secondary ed. }\end{array}$ & $\begin{array}{l}\text { M: part-time } \\
\text { F: full-time }\end{array}$ & $\mathrm{BE}$ & $\mathrm{M}+\mathrm{F}$ \\
\hline Cara & Girl & 4.18 years & 2 parents, 1 younger sister & $\begin{array}{l}\text { M: higher ed. } \\
\text { F: secondary ed. }\end{array}$ & $\begin{array}{l}\text { M: full-time } \\
\text { F: full-time }\end{array}$ & $\mathrm{BE}$ & $\mathrm{M}+\mathrm{F}$ \\
\hline Daisy & Girl & 3.65 years & $\begin{array}{l}2 \text { parents, } 1 \text { older brother and } 1 \text { older } \\
\text { sister (same type of disability) }\end{array}$ & $\begin{array}{l}\text { M: higher ed. } \\
\text { F: higher ed. }\end{array}$ & $\begin{array}{l}\text { M: part-time } \\
\text { F: full-time }\end{array}$ & $\mathrm{BE}$ & M \\
\hline Eva & Girl & 4.98 years & 2 parents, 1 older sister & $\begin{array}{l}\text { M: secondary ed. } \\
\text { F: secondary ed. }\end{array}$ & $\begin{array}{l}\text { M: part-time } \\
\text { F: full-time }\end{array}$ & NL & M \\
\hline Finn & Boy & 4.05 years & 2 parents & $\begin{array}{l}\text { M: higher ed. } \\
\text { F: higher ed. }\end{array}$ & $\begin{array}{l}\text { M: part-time } \\
\text { F: self-employed }\end{array}$ & NL & $\mathrm{M}+\mathrm{F}$ \\
\hline $\begin{array}{l}\text { TD-group } \\
\text { Gwen }\end{array}$ & Girl & 4.01 years & 2 parents, 1 older brother, 1 older sister & $\begin{array}{l}\text { M: higher ed. } \\
\text { F: higher ed. }\end{array}$ & $\begin{array}{l}\text { M: full-time } \\
\text { F: full-time }\end{array}$ & $\mathrm{BE}$ & M \\
\hline Harry & Boy & 3.18 years & 2 parents, 1 younger brother & $\begin{array}{l}\text { M: higher ed. } \\
\text { F: secondary ed. }\end{array}$ & $\begin{array}{l}\text { M: part-time } \\
\text { F: full-time }\end{array}$ & $\mathrm{BE}$ & M \\
\hline Isabel & Girl & 2.43 years & 2 parents & $\begin{array}{l}\text { M: primary ed. } \\
\text { F: higher ed. }\end{array}$ & $\begin{array}{l}\text { M: full-time } \\
\text { F: full-time }\end{array}$ & $\mathrm{BE}$ & $\mathrm{M}+\mathrm{F}$ \\
\hline Jacob & Boy & 3.45 years & 2 parents, 1 younger sister & $\begin{array}{l}\text { M: higher ed. } \\
\text { F: secondary ed. }\end{array}$ & $\begin{array}{l}\text { M: self-employed } \\
\text { F: full-time }\end{array}$ & $\mathrm{BE}$ & M \\
\hline Kevin & Boy & 3.03 years & 2 parents, 1 younger brother & $\begin{array}{l}\text { M: higher ed. } \\
\text { F: primary ed. }\end{array}$ & $\begin{array}{l}\text { M: part-time } \\
\text { F: self-employed }\end{array}$ & $\mathrm{BE}$ & M \\
\hline
\end{tabular}

Note. M stands for mother, F for father. BE stands for Belgium, NL stands for the Netherlands. Child names were altered for confidentiality reasons. 
Running Head: ECOCULTURAL PERSPECTIVE ON FAMILY ACTIVITIES

Table 2

Global rating of the ecological fit, congruence and meaningfulness of each family's daily life routine

\begin{tabular}{llll}
\hline & \multicolumn{3}{c}{ Scores } \\
\cline { 2 - 4 } DD-group & Ecological fit & Congruence & Meaningfulness \\
Arthur & Low & Low & Medium \\
Ben & Medium & Medium & High \\
Cara & High & High & High \\
Daisy & High & High & High \\
Eva & Medium & High & High \\
Finn & Medium & Medium & Medium \\
& & & \\
TD-group & & & \\
Gwen & High & High & High \\
Harry & High & High & High \\
Isabel & High & High & High \\
Jacob & High & High & High \\
Kevin & Low & Low & Low \\
\hline Note. The participants' names were altered for confidentiality reasons
\end{tabular}

Note. The participants' names were altered for confidentiality reasons 
Running Head: ECOCULTURAL PERSPECTIVE ON FAMILY ACTIVITIES

Table 3

Common and DD-specific indicators of ecological fit

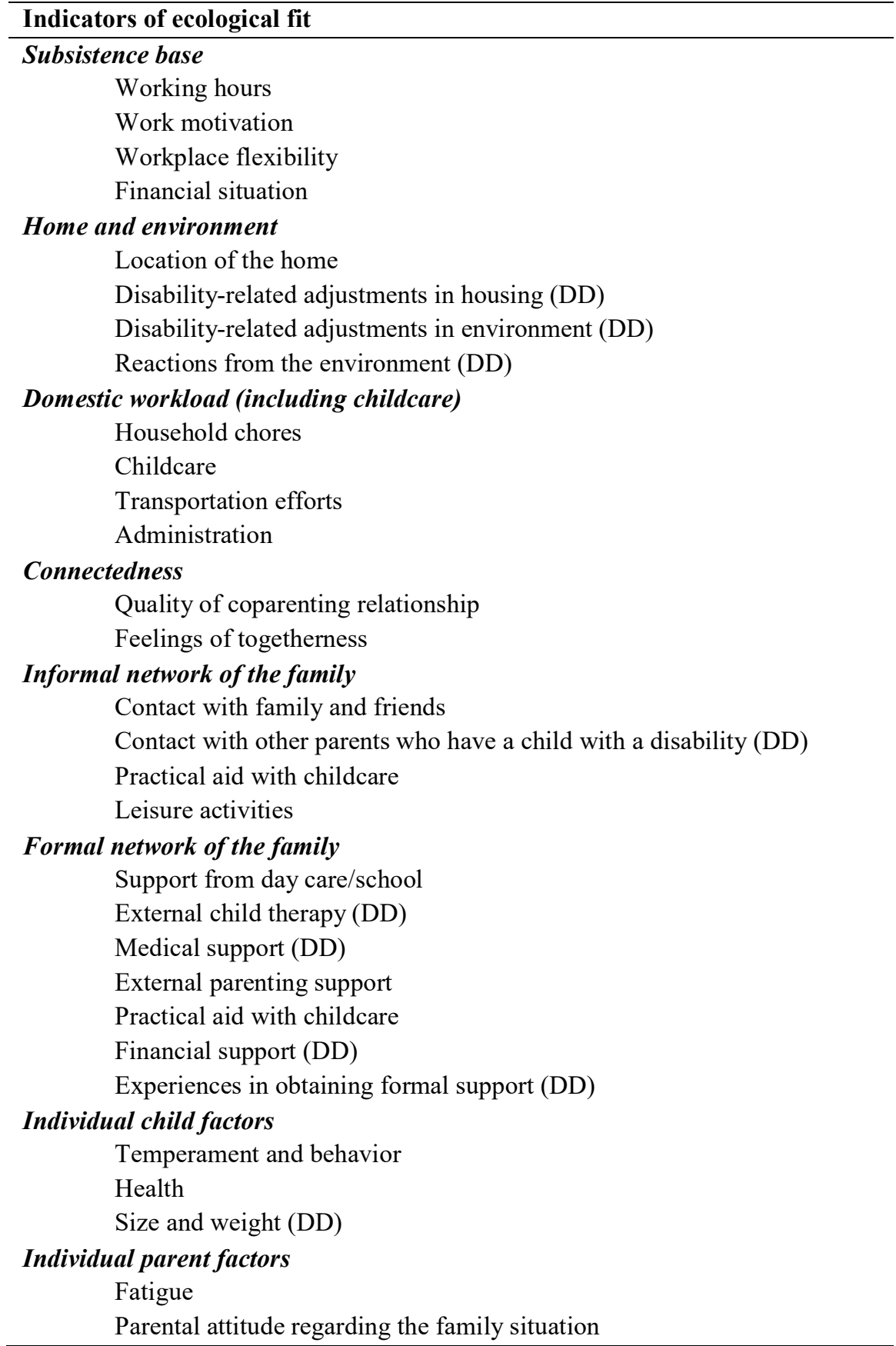

Note: (DD) indicates subdomains that are specific to the DD-group 
Running Head: ECOCULTURAL PERSPECTIVE ON FAMILY ACTIVITIES

Fig. 1

Visualized structure of the administered interview

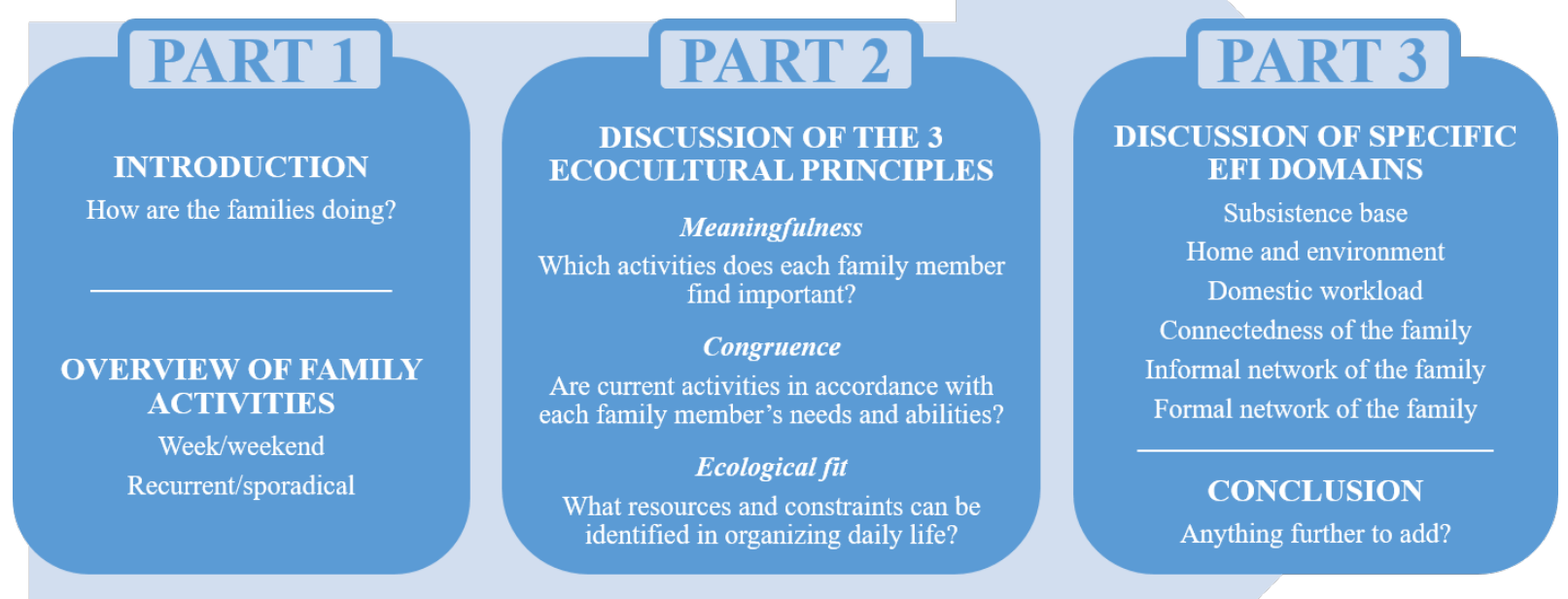

